\title{
Fitting Propagation Models With Random Grains, Method and Some Simulation Studies
}

\author{
M. Khazaei, ${ }^{\dagger}, *$ K. Shafie ${ }^{\ddagger}$ and M. Ganjali ${ }^{\dagger}$ \\ ${ }^{\dagger}$ Shahid Beheshti University \\ ‡ University of Northern Colorado
}

\begin{abstract}
In this paper the regression problem for random sets of the Boolean model type is developed, where the corresponding Poisson process of the model is related to some explanatory variables and the random grains are not affected by these variables. A model we call propagation model, is presented and some methods for fitting this model are introduced. Propagation model is applied in a simulation study.
\end{abstract}

Keywords. Random closed set; hitting functional; Boolean model; propagation model; generalized linear model.

\section{Introduction}

Random set theory was developed by Kendall (1974) and independently by Matheron (1975) based on Choquet's work on capacity functionals. In theory of Matheron (1975) random closed sets (RACS's) in $\mathbb{R}^{d}$ are random elements on the space of closed subsets of $\mathbb{R}^{d}$ which are endowed with hit-or-miss topology. The mathematical foundation of random closed sets is essentially based on Choquet's (capacity) theorem. This theorem characterizes probability distribution of a RACS as a set function called hitting functional. Hitting functional plays the similar role as that of distribution function for random variables and for a RACS $Y$ defined as:

$$
T_{Y}(K)=P_{Y}(Y \cap K \neq \emptyset),
$$

* Corresponding author 
where $K$ is a compact subset in $\mathbb{R}^{d}$.

In this way, probability models were extended for describing the behavior of random phenomena whose outcomes are subsets of $\mathbb{R}^{d}$. Examples of these Phenomena are shape of a tumor or region affected by cancer in medicine, the area hitted by a meteorite or a bomb, the region covered by some plant or fire in a jungle, the activated region of brain by some stimulant and red blood cells in viewing field of a microscope. Boolean model is a parametric model which can generate such random sets. Many characteristics of these models and their statistical analysis can be found in Molchanov (1997), Stoyan et. al. (1995) and Cressie (1993). In section 2 we introduce the Boolean model, its hitting function, some of its related parameters and estimators of these parameters. The regression problem, that is relating the behaviour of the Boolean random sets to some explanatory variables considered in Khazaee and Shafie (2006). They classify these regressions according to the type of explanatory variables as propagation, growth and propagation-growth models. In section 2 we also introduce propagation models for the modelling of the effects of explanatory variable on Boolean random sets. Some methods to fitting propagation models when grains are circles with known radii are presented in Khazaee and Shafie (2006). In this paper we extend the previous work to the case which grains are random radii circles. And in section 3, we present some ideas and methods for fitting this propagation model. In this section we supposed that the grains are random circles with radii having unknown uniform distributions. However, other distributions for radii, e.g. distributions which can be specified with the first two moments, can also be used. This method will be applied in a simulated example in section 4 .

\section{Boolean Model and Propagation Model}

The idea that a random set can be a combination of some simple random sets, has been used in structure of different models of random sets. One of these models which has many abilities in description of behavior of random sets and also modelling the random sets is Boolean model (see Matheron, 1975). A Boolean model is formed by placing random closed sets at the points of a homogeneous Poisson point process $(D)$ and taking the union of these sets. In fact, a Boolean model $Y$ is defined as

$$
Y=\bigcup_{\mathbf{d}_{i} \in D}\left(Z_{i} \oplus \boldsymbol{d}_{i}\right)
$$


where $Z_{i}, i=1,2, \ldots$ are independent copies of random closed set $Z_{0}$ and are independent of $D$. Also $Z \oplus \mathbf{d}=\{\boldsymbol{z}+\boldsymbol{d}: \mathbf{z} \in Z\}$ is Minkowski sum of $Z$ and $\boldsymbol{d}$. The points of the Poisson process are called the germs and the associated random sets the grains. It can be shown (Stoyan et al., 1995) that the hitting functional of a Boolean model is:

$$
T_{Y}(K)=1-\exp \left\{-\lambda E\left[v_{d}\left(Z_{0} \oplus \check{K}\right)\right]\right\}
$$

where $\check{K}=\{-\boldsymbol{k}: \boldsymbol{k} \in K\}, Z_{0} \oplus \check{K}=\bigcup_{\boldsymbol{k} \in K}\left(Z_{0} \oplus(-\boldsymbol{k})\right), v_{d}$ is the Lebesgue measure and $\lambda$ is the intensity of the Poisson process $D$. For a Boolean model with convex grains in $\mathbb{R}^{2}$, it can be shown (see Matheron, 1975) that:

$$
T_{Y}(K)=1-\exp \left\{-\lambda\left[E\left[v_{2}\left(Z_{0}\right)\right]+\frac{1}{2 \pi} E\left[U\left(Z_{0}\right)\right] U(K)+v_{2}(K)\right]\right\},
$$

where $U$ denotes the perimeter. When $Y$ is observed within a window $W$, an unbiased estimator of $T_{Y}(K)$ is obtained by:

$$
\widehat{T}_{Y}(K)=\frac{v_{d}((W \ominus \check{K}) \cap(Y \oplus \check{K}))}{v_{d}(W \ominus \check{K})},
$$

where $W \ominus \check{K}=\bigcap_{\mathbf{k} \in K} W \oplus(-\mathbf{k})$.

The point process of tangent points associated with a Boolean model are used in constructing estimator of $\lambda$ (see Molchanov, 1995). Let assume that the typical grain $Z_{0}$ is almost surely convex. If a direction $u$ in $\mathbb{R}^{d}$ is fixed, then the tangent point of each grain $Z_{i}$ is defined to be the lexicographical minimum among all points at which a hyperplane orthogonal to $u$ which moves in the direction of $u$ first touches $Z_{i}$. Some of these tangent points are covered by other grains while other points are visible. These exposed, or observable, tangent points form a point process $N^{+}(u)$ with intensity $\lambda(1-p)$, where $p$ is the volume fraction of $Y$ (see bellow). If $u$ is directed upwards, then $N^{+}(u)$ is called a lower positive tangent point process. If $n^{+}$is the number of lower positive tangent points in window $W$ then an estimator of $\lambda$ (see Molchanov and Stoyan, 1994) is:

$$
\hat{\lambda}=\frac{n^{+}}{v_{d}(W)(1-\hat{p})}
$$

where $\hat{p}$ is in 5 .

For a Boolean model volume fraction $p$, which is defined as the mean 
fraction of volume occupied by $Y$ in a region of unit volume, is:

$$
p=1-\exp \left\{-\lambda E\left[v_{d}\left(Z_{0}\right)\right]\right\} .
$$

When $Y$ is observed within a window $W$, an unbiased estimator of $p$ is:

$$
\hat{p}=\frac{v_{d}(Y \cap W)}{v_{d}(W)} .
$$

For this and other estimators of $p$ see Stoyan et al. (1995).

Now we consider a situation similar to Figure 1 in which each image is a realization of a Boolean model with a corresponding value for the explanatory variable. The problem is to determine and fit a model for the relationship between the Boolean model and the explanatory variables. In other word, we wish to present a model that is able to generate sets similar to Figure 1 and one can use it for predicting its image by values of an explanatory variable. In this paper we consider the case that the distribution of $Z_{0}$ is independent of explanatory variables and in the next section we shall give a method to fit such a model.

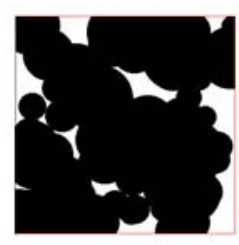

$x=0.95$

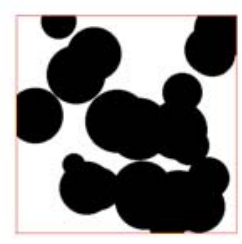

$x=1.23$

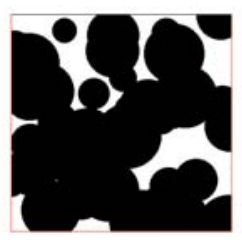

$x=1.03$

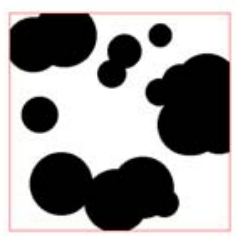

$x=1.37$

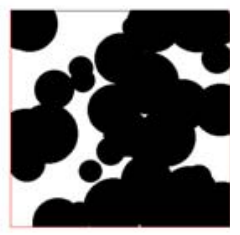

$x=1.12$

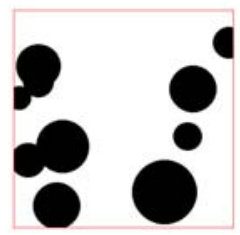

$x=1.47$

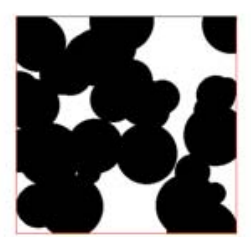

$$
x=1.18
$$

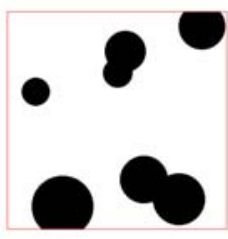

$x=1.68$

Figure 4. Realizations of the Boolean models with 8 values of propagation variable $x$, where the grains are random circles. 
Let $X=\left(x_{1}, x_{2}, \ldots, x_{k}\right)^{\prime}$ be a vector of explanatory variables which we want to use in modelling its relation with the Boolean random set $Y$ and to evaluate its effect on $Y$. In this paper we suppose that $X$ only has some effects on $D$. We shall call such explanatory variables the propagation variables and define a propagation model as:

$$
\left\{\begin{array}{l}
Y_{X}=\bigcup_{\boldsymbol{d}_{i} \in D_{X}}\left(Z_{i} \oplus \boldsymbol{d}_{i}\right), \\
\lambda_{X}=f(X, \boldsymbol{\beta})>0, \\
Z_{i}^{\prime} \text { s are i.i.d. copies of } Z_{0} \text { and are independent of } D_{X} .
\end{array}\right.
$$

where $f$ determines the relation between $X$ and $\lambda$ (the only parameter of $D$ ) and $\boldsymbol{\beta}$ is the parameter for this relationship.

\section{Fitting Methods}

In this section, we present an approach to fit the propagation model (6). For simplicity we suppose $f(X, \boldsymbol{\beta})=h\left(X^{\prime} \boldsymbol{\beta}\right)$, where $h$ is a differentiable monotone positive function and $Z_{0}$ is a circle with radius $R$ where $R$ is uniformly distributed on $(a, b)$. This approach can be also extended for any distribution of $R$ which is characterized with first two moments. Under these conditions propagation model (6) has the following form:

$$
\left\{\begin{array}{l}
Y_{X}=\bigcup_{\boldsymbol{d}_{i} \in D_{X}}\left(Z_{i} \oplus \boldsymbol{d}_{i}\right), \\
\lambda_{X}=f(X, \boldsymbol{\beta})=h\left(X^{\prime} \boldsymbol{\beta}\right), \\
Z_{0}=B(\mathbf{o}, R), \text { where } R \sim \text { uniform }(a, b), \\
\text { and is independent of } D_{X} .
\end{array}\right.
$$

Figure 1 shows a realization of this propagation model with 8 values of propagation variable $x$ where $x$ is the only explanatory variable and for $i=1,2, \ldots, 8, f\left(x_{i}, \boldsymbol{\beta}\right)=\exp \left(\beta_{0}+\beta_{1} x_{i}\right)=\exp \left(7.1-3 x_{i}\right)$ and $R_{i} \sim$ uniform $(0.05,0.15)$.

In the following we shall estimate the $\boldsymbol{\beta}, a$ and $b$ i.e., parameters of the propagation model (7).

Estimation of $\boldsymbol{\beta}$ : When $R$ is constant three methods for estimating of $\boldsymbol{\beta}$ are given in Khazaee and Shafie (2006). All of these methods can also be applied for our case. In this paper, we used method I. Let $n_{i}$ be the number of points 
in $D_{X_{i}}$ in a window $W_{i}$. We know that $n_{i}$ follows a Poisson distribution with the mean $\lambda_{i}=f\left(X_{i}, \boldsymbol{\beta}\right)=h\left(X_{i}^{\prime} \boldsymbol{\beta}\right)$. Now, we can say that the problem of estimating $\boldsymbol{\beta}$ is a problem of estimating the parameters of a generalized linear model with Poisson family, where the link function is $g(\cdot)=h^{-1}(\cdot)$. The maximum likelihood estimate for $\boldsymbol{\beta}$ can be computed using iterative reweighted least squares algorithm for fitting a linear regression model, $z_{i}=$ $X_{i}^{\prime} \boldsymbol{\beta}+\epsilon_{i}$, where

$$
z_{i}=g\left(\lambda_{i}\right)+\left(n_{i}-\lambda_{i}\right) g^{\prime}\left(\lambda_{i}\right),
$$

and the weights are $w_{i}=\left[\operatorname{var}\left(z_{i}\right)\right]^{-1}=\left[\operatorname{var}\left(n_{i}\right)\right]^{-1}\left[g^{\prime}\left(\lambda_{i}\right)\right]^{-2}$ (for more explanation about this algorithm and its convergence see McCullagh and Nelder, 1989). To implement this algorithm, we use $\hat{\lambda}_{i}^{(0)}$ 's as initial estimates for $\lambda_{i}$ 's (usually $\hat{\lambda}_{i}^{(0)}=n_{i}$ ). Then, the values of dependent variable and weights can be computed by $z_{i}^{(0)}=g\left(\hat{\lambda}_{i}^{(0)}\right)+\left(n_{i}-\hat{\lambda}_{i}^{(0)}\right) g^{\prime}\left(\hat{\lambda}_{i}^{(0)}\right)$ and $w_{i}^{(0)}=1 /\left\{\hat{\lambda}_{i}^{(0)}\right\}$ $\left[g^{\prime}\left(\hat{\lambda}_{i}^{(0)}\right)\right]^{-2}$, respectively. Let $Z^{(0)}=\left(z_{1}^{(0)}, \ldots, z_{n}^{(0)}\right), \boldsymbol{w}^{(0)}$ be a diagonal matrix with the diagonal elements of $w_{i}^{(0)}$ and $\boldsymbol{X}$ be the observed matrix of the explanatory vector $X$. The first approximation of $\hat{\boldsymbol{\beta}}, \hat{\boldsymbol{\beta}}^{(1)}$, will be obtained by solving the following equation:

$$
\left(\boldsymbol{X}^{\prime} \boldsymbol{w}^{(0)} \boldsymbol{X}\right) \hat{\boldsymbol{\beta}}^{(1)}=\boldsymbol{X}^{\prime} \boldsymbol{w}^{(0)} Z^{(0)} .
$$

Then for $i=1,2, \ldots, n, \hat{\lambda}_{i}^{(1)}=h\left(X_{i}^{\prime} \hat{\boldsymbol{\beta}}^{(1)}\right)$ are calculated and the second iteration can be followed in a similar manner. The procedure will be continued until the convergence to $\hat{\boldsymbol{\beta}}$ occurs. It can be shown (see McCullagh and Nelder, 1989) that if $\boldsymbol{w}$ is the weights matrix in the last step, then the approximate distribution for $\hat{\boldsymbol{\beta}}$ is,

$$
N\left(\boldsymbol{\beta},\left(\boldsymbol{X}^{\prime} \boldsymbol{w} \boldsymbol{X}\right)^{-1}\right) .
$$

There is no observable $n_{i}$ for the overlapped grains, but instead it we used a suitable estimate in computational algorithm, for example, by using (5):

$$
\hat{n}_{i}=\left[v_{2}\left(W_{i}\right) \hat{\lambda}_{i}\right]=\left[\frac{n_{i}^{+}}{1-\hat{p}_{i}}\right],
$$

where $n_{i}^{+}$is the number of lower positive tangent points in the window $W_{i}$, and $\hat{p}_{i}$ is an estimate of volume fraction for $i$ th Boolean model obtained from (5).

Estimation of $\boldsymbol{a}$ and $\boldsymbol{b}$ : In order to estimate $a$ and $b$ we use intensity 
(or moment) method. In this method the relation between parameters will be used. The estimates of parameters, which can be directly calculated by available realizations, will be substituted in these equations. Then the given equations will be solved for other parameter estimates. Here, as $R \sim$ uniform $(a, b)$ then these equation are:

$$
\left\{\begin{array}{l}
a=E(R)-\sqrt{3}\left(E\left(R^{2}\right)-E(R)^{2}\right)^{1 / 2} \\
b=E(R)+\sqrt{3}\left(E\left(R^{2}\right)-E(R)^{2}\right)^{1 / 2} .
\end{array}\right.
$$

So if $\widehat{E\left(R^{2}\right)}$ and $\widehat{E(R)}$ are suitable estimators of $E\left(R^{2}\right)$ and $E(R)$, then the intensity method estimators of $a$ and $b$ are:

$$
\left\{\begin{aligned}
\hat{a} & =\widehat{E}(R)-\sqrt{3}\left(\widehat{E}\left(R^{2}\right)-\widehat{E}(R)^{2}\right)^{1 / 2} \hat{b} \\
& =\widehat{E}(R)+\sqrt{3}\left(\widehat{E}\left(R^{2}\right)-\widehat{E}(R)^{2}\right)^{1 / 2}
\end{aligned}\right.
$$

Our approach for estimation of $E\left(R^{2}\right)$ is using equation (4) with $E\left[v_{2}\left(Z_{0}\right)\right]$ $=\pi E\left(R^{2}\right)$. From this equation we have

$$
E\left(R^{2}\right)=-\frac{\ln (1-p)}{\pi \lambda} .
$$

For $i=1,2, \ldots, n$ we substitute $\hat{p}_{i}$ and $\hat{\lambda}_{i}$ in above equation to find an estimator of $E\left(R^{2}\right)$ for each realization. We show these estimators with $\widehat{E}_{i}\left(R^{2}\right)$ and use the mean of these estimators for estimation of $E\left(R^{2}\right)$, i.e:

$$
\widehat{E}\left(R^{2}\right)=\frac{1}{n} \sum_{i=1}^{n} \widehat{E}_{i}\left(R^{2}\right)=-\frac{1}{n} \sum_{i=1}^{n} \frac{\ln \left(1-\hat{p}_{i}\right)}{\pi \hat{\lambda}_{i}} .
$$

In order to estimate $E(R)$ we use the equation (1). When the grains are random circles and we choose $K_{t}$ as a circle with radius $t$ and center origin, this equation changes to the following form

$$
Q_{Y}\left(K_{t}\right)=1-T_{Y}\left(K_{t}\right)=\exp \left\{-\lambda\left[\pi E\left(R^{2}\right)+2 \pi t E(R)+\pi t^{2}\right]\right\} .
$$

From this equation moment method estimator of $E_{i}(R)$ is

$$
\widehat{E}_{i}(R)=\frac{1}{2 \pi t}\left[-\frac{\ln \widehat{Q}_{Y_{i}}\left(K_{t}\right)}{\hat{\lambda}_{i}}-\pi \widehat{E}_{i}\left(R^{2}\right)-\pi t^{2}\right] .
$$


We use the mean of these estimators for estimating of $E(R)$, i.e,:

$$
\widehat{E}(R)=\frac{1}{n} \sum_{i=1}^{n} \widehat{E}_{i}(R)=\frac{1}{2 \pi t n} \sum_{i=1}^{n}\left[-\frac{\ln \widehat{Q}_{Y_{i}}\left(K_{t}\right)}{\hat{\lambda}_{i}}-\pi \widehat{E}_{i}\left(R^{2}\right)-\pi t^{2}\right],
$$

where for $i=1,2, \ldots, n, \widehat{Q}_{Y_{i}}\left(K_{t}\right)=1-\widehat{T}_{Y_{i}}\left(K_{t}\right)$ can be calculated from (2).

\section{Application and Simulation Studies}

For a better explanation of this we fit the following model to simulate observations in Figure 1.

$$
Y_{X}=\bigcup_{\boldsymbol{d}_{i} \in D_{X}}\left(Z_{i} \oplus \boldsymbol{d}_{i}\right)
$$

where $D_{x}$ is a Poisson point process with intensity $\lambda=\exp \left(\beta_{0}+\beta_{1} x\right)$ and $Z_{i}$ 's are i.i.d copies of a random circle where its radius is distributed uniformly on $(a, b)$.

Estimation of $\boldsymbol{\beta}$ : For estimation of $\boldsymbol{\beta}$ we computed the statistics in Table 1 by using (5) and (9). Using method I described in previous section, estimates of $\beta_{0}, \beta_{1}$ and their standard errors are given in Table 2 .

Estimation of $\boldsymbol{a}$ and $\boldsymbol{b}$ : In our example values of $\widehat{E}_{i}\left(R^{2}\right)$ have been obtained by substituting $\hat{p}_{i}$ and $\hat{\lambda}_{i}$ from Table 1 in (11) and are given in Table 2, thus from (12) we have $\widehat{E}\left(R^{2}\right)=0.0116$.

Figure 2 illustrates the way $\widehat{Q}_{Y_{7}}\left(K_{t}\right)$ is calculated. Table 3 shows the values of $\widehat{Q}_{Y_{i}}\left(K_{t}\right)$, calculated from each realizations in Figure 1 for $t=0.01$, and values of $\widehat{E}_{i}(R)$ obtained from (13). From (14), $\widehat{E}(R)=0.1034$. Finally put $\widehat{E}(R)=0.1034$ and $\widehat{E}\left(R^{2}\right)=0.0116$ in $(10)$ we get $\hat{a}=0.052$ and $\hat{b}=0.156$. Therefore the fitted model to the observations in Figure 1 is

$$
\widehat{Y}_{x}=\bigcup_{\boldsymbol{d}_{i} \in \widehat{D}_{x}}\left(Z_{i} \oplus \boldsymbol{d}_{i}\right)
$$

where $\widehat{D}_{x}$ is a Poisson point process with intensity $\hat{\lambda}=\exp (7.31-3.27 x)$ and $Z_{i}$ 's are i.i.d copies of a random circle with a radius is distributed uniformly on $(0.052,0.156)$. 
Table 1.statistics obtained from Figure 1

\begin{tabular}{ccccccccc}
\hline \hline$i$ & 1 & 2 & 3 & 4 & 5 & 6 & 7 & 8 \\
\hline$x$ & 0.95 & 1.03 & 1.12 & 1.18 & 1.23 & 1.37 & 1.47 & 1.68 \\
$n^{+}$ & 9 & 11 & 11 & 9 & 8 & 12 & 7 & 6 \\
$\hat{p}$ & 0.87 & 0.79 & 0.73 & 0.73 & 0.57 & 0.46 & 0.39 & 0.28 \\
$\hat{n}=\hat{\lambda}$ & 69 & 52 & 40 & 33 & 19 & 22 & 11 & 6 \\
\hline
\end{tabular}

Table 2. Estimates of parameters and their standard errors.

\begin{tabular}{ccc}
\hline \hline$i$ & $\hat{\beta}_{i}$ & s.e $\left(\hat{\beta}_{i}\right)$ \\
\hline 0 & 7.312 & 0.8442 \\
1 & -3.274 & 0.5625 \\
\hline
\end{tabular}

Table 3. Estimates of $Q_{Y}\left(K_{0.01}\right), E\left(R^{2}\right)$ and $E(R)$ for $i$ th realization in Figure 1.

\begin{tabular}{ccccccccc}
\hline \hline$i$ & 1 & 2 & 3 & 4 & 5 & 6 & 7 & 8 \\
\hline$\widehat{Q}_{Y_{i}}\left(K_{0.01}\right)$ & 0.08 & 0.14 & 0.22 & 0.23 & 0.36 & 0.47 & 0.58 & 0.75 \\
$\widehat{E}_{i}\left(R^{2}\right)$ & 0.0094 & 0.0096 & 0.0104 & 0.0126 & 0.0141 & 0.0089 & 0.0143 & 0.0132 \\
$\widehat{E}_{i}(R)$ & 0.108 & 0.119 & 0.077 & 0.116 & 0.144 & 0.096 & 0.068 & 0.099 \\
\hline
\end{tabular}

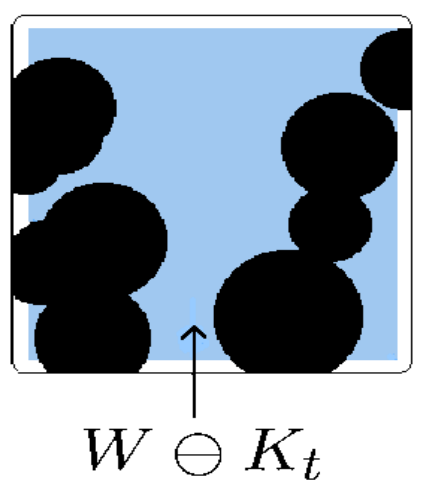

Figure 5. An illustration of the way $\widehat{Q}_{Y_{7}}\left(K_{0.01}\right)$ is computed. The black region is $Y \oplus K_{0.01}$ and $\widehat{T}_{Y_{7}}\left(K_{0.01}\right)=1-\widehat{Q}_{Y_{7}}\left(K_{0.01}\right)$ is proportion of this region in window $W \ominus K_{0.01}$. 
Table 4. mean and standard deviation of the simulation estimates

\begin{tabular}{ccccccc}
\hline \hline & $\hat{\beta}_{0}$ & $\hat{\beta}_{1}$ & $\hat{a}$ & $\hat{b}$ & $\widehat{E(R)}$ & $\widehat{E\left(R^{2}\right)}$ \\
\hline mean & 6.84 & -2.89 & 0.083 & 0.139 & 0.1111 & 0.0117 \\
std.dev & 0.935 & 0.742 & 0.0098 & 0.0178 & 0.0066 & 0.0018 \\
\hline
\end{tabular}

For examining properties of estimators, $\hat{\boldsymbol{\beta}}, \hat{a}$ and $\hat{b}$ results of a simulation study are given in the following. For this we simulate 1000 times 8 realizations from model in example 1 and calculate $\hat{\beta}_{0}, \hat{\beta}_{1}, \hat{a}$ and $\hat{b}$. Table 4 shows the mean and standard deviation of these estimates. With comparison of mean $\left(\hat{\beta}_{0}\right)=6.84$ and mean $\left(\hat{\beta}_{1}\right)=-2.89$ with their true values $\beta_{0}=7$ and $\beta_{1}=-3$, percent of relative absolute value of bias of estimators $\hat{\beta}_{0}$ and $\hat{\beta}_{1}$ are respectively, $\% 3.7$ and $\% 2.3$. Due to the small sample size $(n=8)$, this does not show considerable differences with its asymptotic values given in (8). To study the performance of the values of asymptotic standard error of $\hat{\beta}_{0}$ and $\hat{\beta}_{1}$ these values are calculated from asymptotic distribution (8) in each iteration. Mean of these values are respectively, mean $\left(\operatorname{s.e}\left(\hat{\beta}_{0}\right)\right)=0.861$ and mean $\left(s . e\left(\hat{\beta}_{1}\right)\right)=0.614$. Difference of these values with $s t d . d e v\left(\hat{\beta}_{0}\right)$ and std.dev $\left(\hat{\beta}_{1}\right)$ given in the table 4 is due to the small sample size. Furthermore, histograms and Q-Q plots of $\hat{\beta}_{0}$ and $\hat{\beta}_{1}$ are shown in Figure 3. These
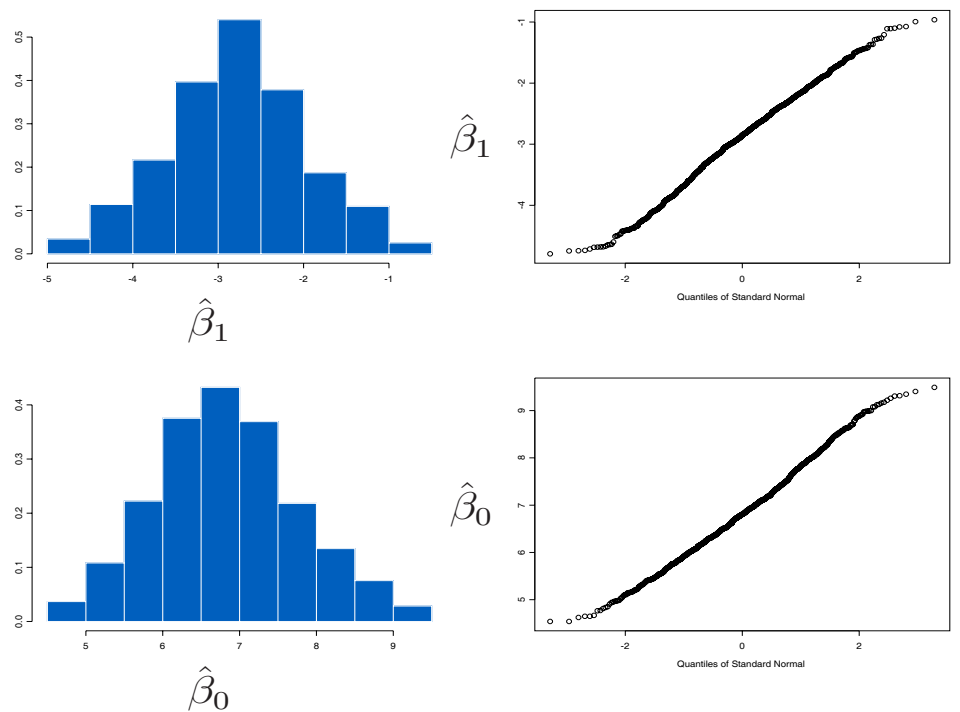

Figure 6. Histograms and Q-Q plots of $\hat{\beta}_{0}$ and $\hat{\beta}_{1}$ 
show that normal distributions are good approximations for distributions of $\hat{\beta}_{0}$ and $\hat{\beta}_{1}$. In general, above results show that asymptotic distribution (8) provides a good approximate for distribution of $\hat{\boldsymbol{\beta}}$. Regarding parameter estimates of radius distribution i.e. $a$ and $b$, bias values are unfortunately considerable, especially for estimating parameter $a$. We think that these biases come from the estimates used for $E(R)$ and $E\left(R^{2}\right)$, especially estimate used for $E(R)$. Mean of estimates values of simulation for $E(R)$ and $E\left(R^{2}\right)$ are also calculated and are given in table 4 . In comparison of these values with the true values of $E(R)=0.1$ and $E\left(R^{2}\right)=0.0108$, percents of relative absolute values of bias are \%11.1 and \%8.3, respectively. There is another problem with estimates (14) for $E(R)$. Roughly in $\% 7$ of simulations values of $\hat{E}(R)^{2}$ are greater than $\hat{E}\left(R^{2}\right)$ and so estimations of $a$ and $b$ in (10) are not possible. In this simulation study, we delete these cases. One possible future research is to find better estimators for $E(R)$.

\section{Conclusion}

This paper illustrated that propagation models which can be applied for modelling the relationship between Boolean random sets and covariates. In this paper, extracted statistics from realization of Boolean model, which can be applied in fitting propagation models, were presented and a method for fitting propagation model was introduced. Our study showed that the presented estimation method is good to be used, but the need for finding a better estimator for $E(R)$ may be regarded as a new research. Also, on selection and goodness of fit of the model more works need to be done.

\section{References}

Cressie, N. (1993). Statistics for Spatial Data. Wiley, New York.

Khazaee, M. and Shafie, K. (2006). Regression models for the Boolean random sets. J. Appl. Stat. 33, 557-567.

Kendall, D.G. (1974). Foundations of a Theory of Random Sets. Stochastic Geometry, ed. E. F. Harding and D. G. Kendall, 332-376, Wiley, Chichester.

Matheron, G. (1975). Random Sets and Integral Geometry. Wiley, New York.

McCullagh, P. and Nelder, J.A. (1989). Generalized Linear Model. Chapman \& Hall, London.

Molchanov, I.S. and Stoyan, D. (1994). Asymptotic properties of estimators for parameters of the Boolean model. Adv. Appl. Prob. 26, 301-323. 
Molchanov, I.S. (1997). Statistics of the Boolean Model for Practitioners and Mathematicians. Wiley, Chichester.

Stoyan, D.; Kendall, W.S. and Mecke, J. (1995). Stochastic Geometry and Its Applications. Wiley, Chichester.

\section{Mojtaba Khazaei}

Department of Statistics, Faculty of Mathematical Sciences, Shahid Beheshti University, Tehran, Iran. e-mail:m_khazaei@sbu.ac.ir

\section{Mojtaba Ganjali}

Department of Statistics, Faculty of Mathematical Sciences, Shahid Beheshti University, Tehran, Iran.

e-mail:m-ganjali@sbu.ac.ir

\section{Khalil Shafie}

Applied Statistics and Research Methods, University of Northern Colorado,

Colorado, USA.

e-mail:khalil.shafie@unco.edu 